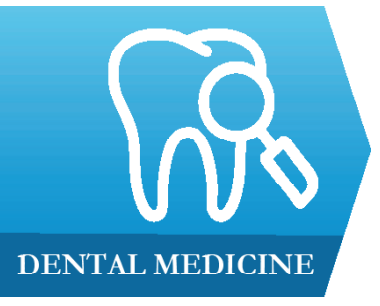

1) Dental Prevention Department, Faculty of Dental Medicine, Iuliu Hatieganu University of Medicine and Pharmacy, Cluj-Napoca, Romania

2) Discipline of Anatomy and Embryology, Faculty of General Medicine, Iuliu Hatieganu University of Medicine and Pharmacy, ClujNapoca, Romania

3) Computer Science Department, Technical University of Cluj-Napoca, Romania

4) Department of Histology, Iuliu Hatieganu University of Medicine and Pharmacy, Cluj-Napoca, Romania

DOI: $10.15386 / \mathrm{mpr}-1521$

Manuscript received: 23.07.2019

Received in revised form: 16.09.2019 Accepted: 30.09.2019

Address for correspondence:

chiforradu@gmail.com

\title{
Periodontal evaluation using a non-invasive imaging method (ultrasonography)
}

\author{
Radu Chifor ${ }^{1}$, Alexandru Florin Badea ${ }^{2}$, Ioana Chifor ${ }^{1}$, \\ Delia-Alexandrina Mitrea ${ }^{3}$, Maria Crisan ${ }^{4}$, Mindra Eugenia Badea ${ }^{1}$
}

\begin{abstract}
The periodontal disease and gingival bleeding are highly prevalent in the adult population worldwide. The World Health Organization (WHO) data shows that $90-100 \%$ of the 34 -year-old adults present gingival inflammation. Therefore, an investigation method is required to allow the assessment of the periodontal disease as well as the monitoring of the evolution of the gingival inflammation after periodontal treatments. Non-invasive and operator-independent methods for periodontal examination are necessary for diagnosing and monitoring the periodontal disease. The periodontal ultrasonography is a reliable technique for visualizing the anatomical elements which are necessary to diagnose the periodontal status. Using this imaging technique the dentino-enamel junction, the cortical bone, the radicular surface from the crown to the alveolar bone, the gingival tissue can be seen without interfering with those elements during the examination. Also, calculus visualization is possible before and after scaling in order to evaluate the quality of the treatment.

Using 2D ultrasonography is not feasible in dental practice as it requires extensive experience and is also time consuming. The reproducibility of the $2 \mathrm{D}$ slices is very difficult in order to have the possibility to compare different investigations efficiently. 3D reconstructions of the periodontal tissue can be a very good alternative to eliminate the operator dependence.

Ultrasonography allows the practitioner to visualize the anatomic elements involved in making a periodontal diagnosis. It also allows tracking of subsequent changes. This method is not commonly used for periodontal examination and further studies are required. Previous studies show that ultrasonography can be a reliable noninvasive method to diagnose and monitor the periodontal disease.
\end{abstract}

Keywords: periodontal ultrasonography, non-invasive imaging method, tridimensional imaging method, soft tissue imaging, periodontal diagnosis

\section{Introduction}

The periodontal disease and gingival bleeding are highly prevalent in the adult population worldwide. The World Health Organization (WHO) data shows that $90-100 \%$ of the 34 -year-old adults present gingival inflammation [1]. Percentages of $10-15 \%$ of the adult population all over the world suffer from advanced periodontal disease with at least 1 periodontal pocket higher than $6 \mathrm{~mm}[1]$.

Early action is needed because places where there is gingival bleeding on probing, present a $70 \%$ higher likelihood of gingival attachment loss, in comparison to places with no gingival bleeding on probing. Therefore, Lang et al. concluded that persistence of gingivitis is a risk factor in the loss of periodontal attachment, followed by the loss of teeth due to the increase in mobility [2]. The gingival margin has an important role in the protection of the underlying tissues and in supporting the teeth and implants. The oral health is affected if the gingiva's adhesion to the tooth is lost, through the means of the junctional epithelium, and consequently periodontal diseases occur [3]. 
Other studies show almost the same data and highlight the importance of the early detection and diagnosis. Gingivitis and chronic periodontitis are highly prevalent chronic inflammatory diseases. Gingivitis affects most people, while advanced periodontitis is estimated to affect $5-15 \%$ of adults. The detection and diagnosis of these common diseases is a fundamentally important component of oral health care [4].

The periodontal probe is an instrument in dentistry commonly used during periodontal probing, a clinical procedure that allows the clinician to evaluate the periodontal pocket depth. The periodontal probe remains the primary diagnostic tool and is used to detect the presence of periodontal pockets as measured from the gingival margin to the base of the crevice and the loss of attachment as measured from the cemento-enamel junction to the base of the crevice. The measurements recorded by the probe, however, are not in fact the actual pocket depth or attachment level but the distance from a fixed reference point to where the probe tip penetrates the tissues. This measurement will depend upon the probing pressure used, the tine size of the probe tip, the angulation of the probe, the presence of subgingival deposits and, most importantly, the presence or absence of inflammation in the tissues [5].

In order to reduce the operator dependence computer aided automated probing of periodontal pockets can be used. Studies show that the cemento-enamel junction (CEJ) probe has proved to have the greatest potential for accuracy and reliability for the measurements of clinical attachment level than the Florida stent probe (FSP) and manual probe (UNC-15), and FSP has proved to be better than manual probe (UNC-15), indicating that automated probes are better as far as accuracy, consistency, and reliability are concerned for the measurements of attachment levels [6].

Radiological examinations are complementary methods to diagnose and monitor periodontitis. The most commonly used X-rays in the diagnosis of deep periodontal diseases are the two-dimensional periapical radiographs for the frontal teeth and the bitewing for the lateral teeth [7].

Therefore, an investigation method which allows the assessment of periodontal disease as well as the monitoring of the evolution of the gingival inflammation after periodontal treatments is required.

Non-invasive and operator independent methods of periodontal examination are necessary for diagnosing and monitoring the periodontal disease.

It is natural to think about leveraging the most recent developments in hi-tech in order to overcome the inaccuracy, tissue trauma that characterize the probing methodology currently used in practice.

Bidimensional ultrasonography has all the characteristics mentioned previously, but it is not used daily on large scale in dentistry. In other fields of medicine, ultrasonography is a reliable imaging technique.

We propose tridimensional ultrasonography as an imaging examination technique which is non-invasive, cost-effective, highly accurate, operator independent and allows tracking over time.

\section{Brief main headings}

In the beginning it was necessary to demonstrate that the periodontal ultrasound examinations were reliable imaging techniques. Like any research in medical fields the first studies are more efficient and without any risk if they are performed on experimental models, in vitro.

There are two main objectives to be followed:

the anatomical elements analyzed for periodontal diagnosis can be visualized during the investigations

The accuracy of the measurements performed on the obtained data

Because of the anatomical particularities and the high costs of the devices in this research stage the authors performed the studies using 2D ultrasound machines. The 3D transducers are larger than bidimensional ones and during the examinations the curvatures of the maxilla and of the mandible do not permit a proper contact with the transducer which has a large footprint.

\section{Bidimensional periodontal ultraonographic investigations, using 2D linear transducers on a general-purpose ultrasound machine}

Measuring and characterization of the dentoalveolar system using high frequencies ultrasound techniques (Figure 1). Experimental models, pig mandibles, in vitro study (Figure 2)

Using high frequency ultrasonography, it is possible to assess the periodontal system because all the anatomical elements needed for the diagnosis can be visualized (Figures 3, 4, 5). The method offers quantitative information, precise and repeatable measurements of different areas of the periodontium, which means that the imaging ultrasound method could have clinical applications in periodontology [8].

A second study on an experimental model compared $20 \mathrm{MHz}$ periodontal ultrasonography with periodontal computed tomography scans having as a gold standard the direct microscopy for the same sections obtained through the periodontal system (Figure 6). 


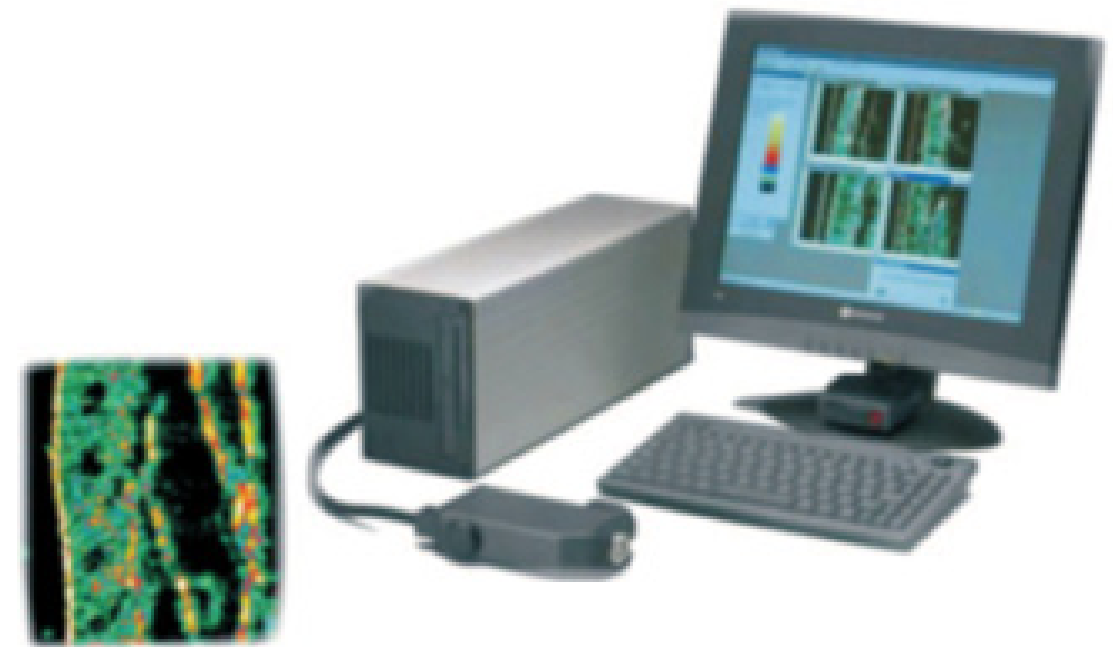

Figure 1. Dermascan C, CORTEX TECHNOLOGY, $20 \mathrm{MHz}, 2 \mathrm{D}$ transductor, Sweden.

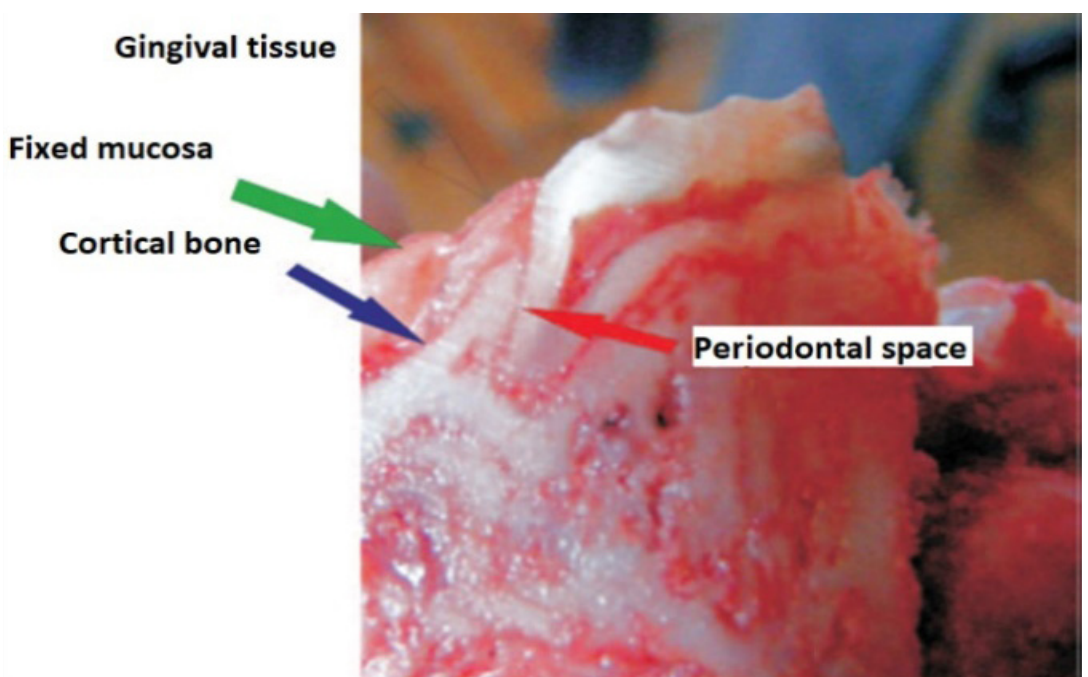

Figure 2. Pig tooth and periodontal tissue - longitudinal section using Buehler microtom, frontal plane [8].
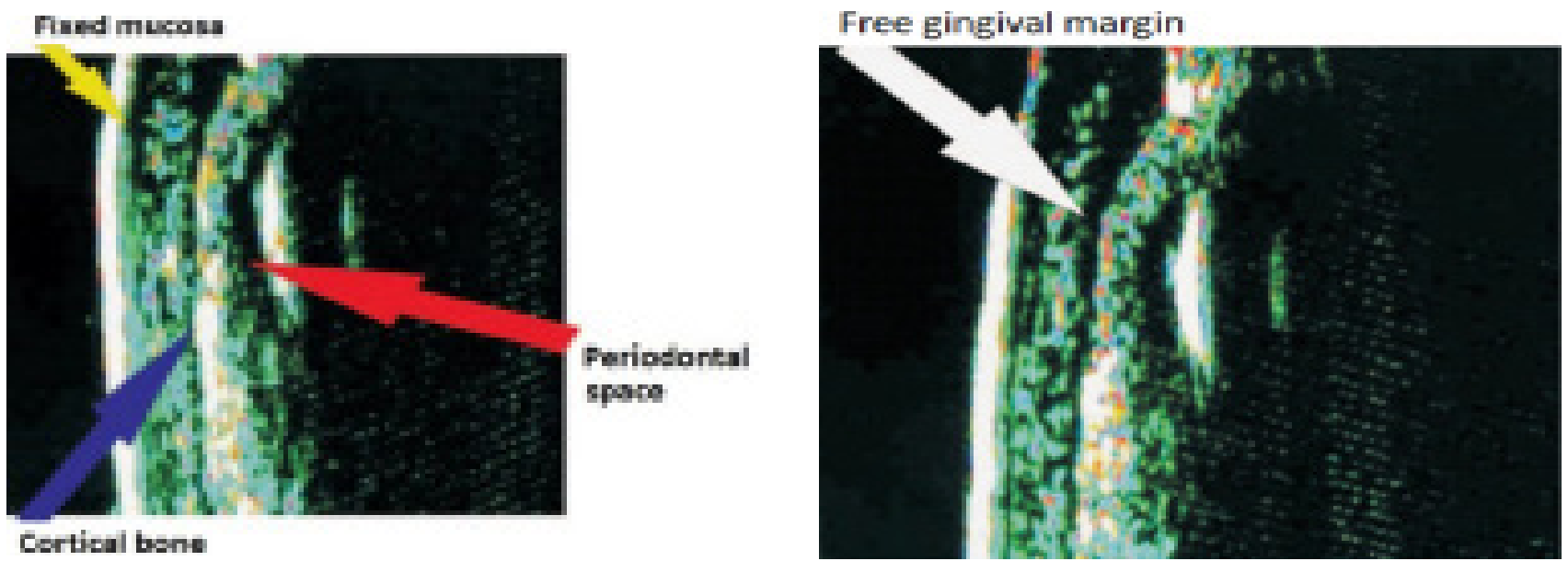

Certical bone

Figures 3, 4. Identification of anatomical references for periodontal diagnosis using 2D high ultrasound examinations: cortical bone, tooth crown, tooth root [8]. 


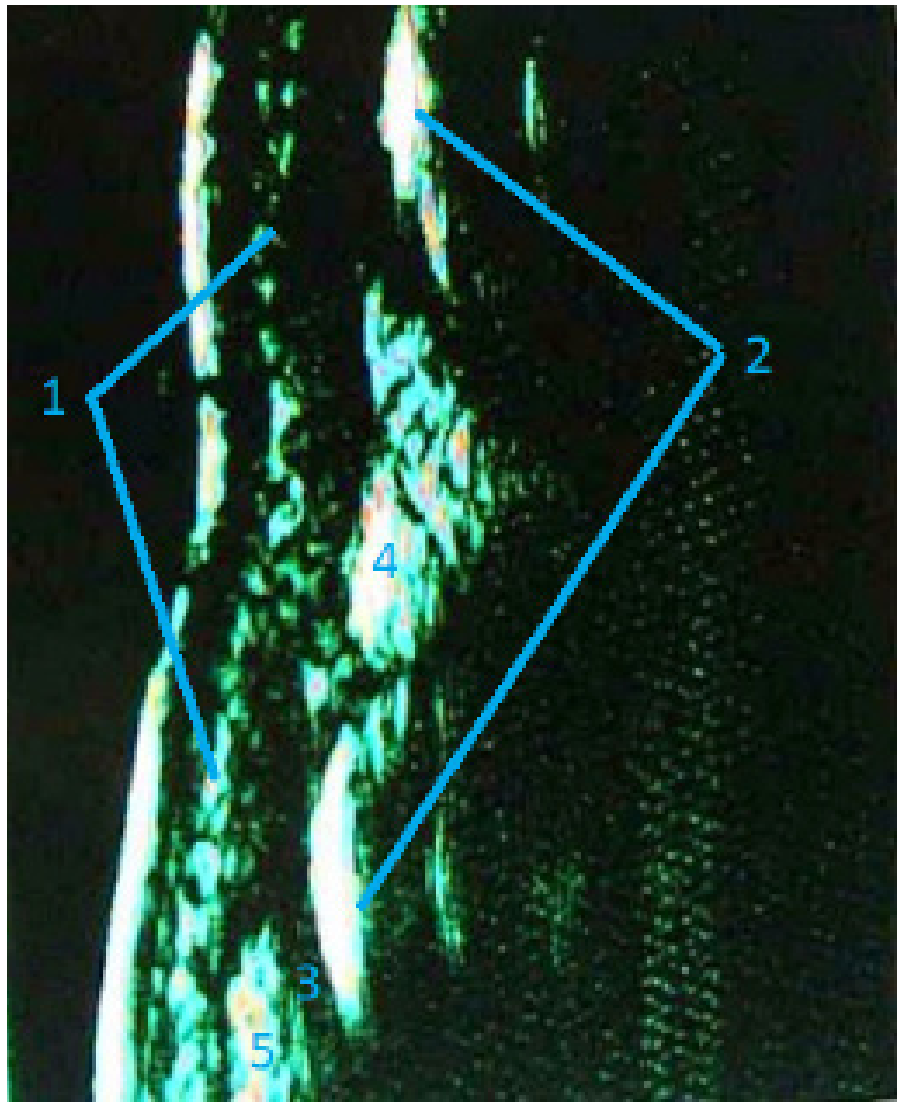

Figure 5. Cross-section, visualized anatomical elements: 1- fixed gingiva; 2- tooth root; 3- periodontal space; 4- interdental cortical bone; 5- external cortical bone [8].
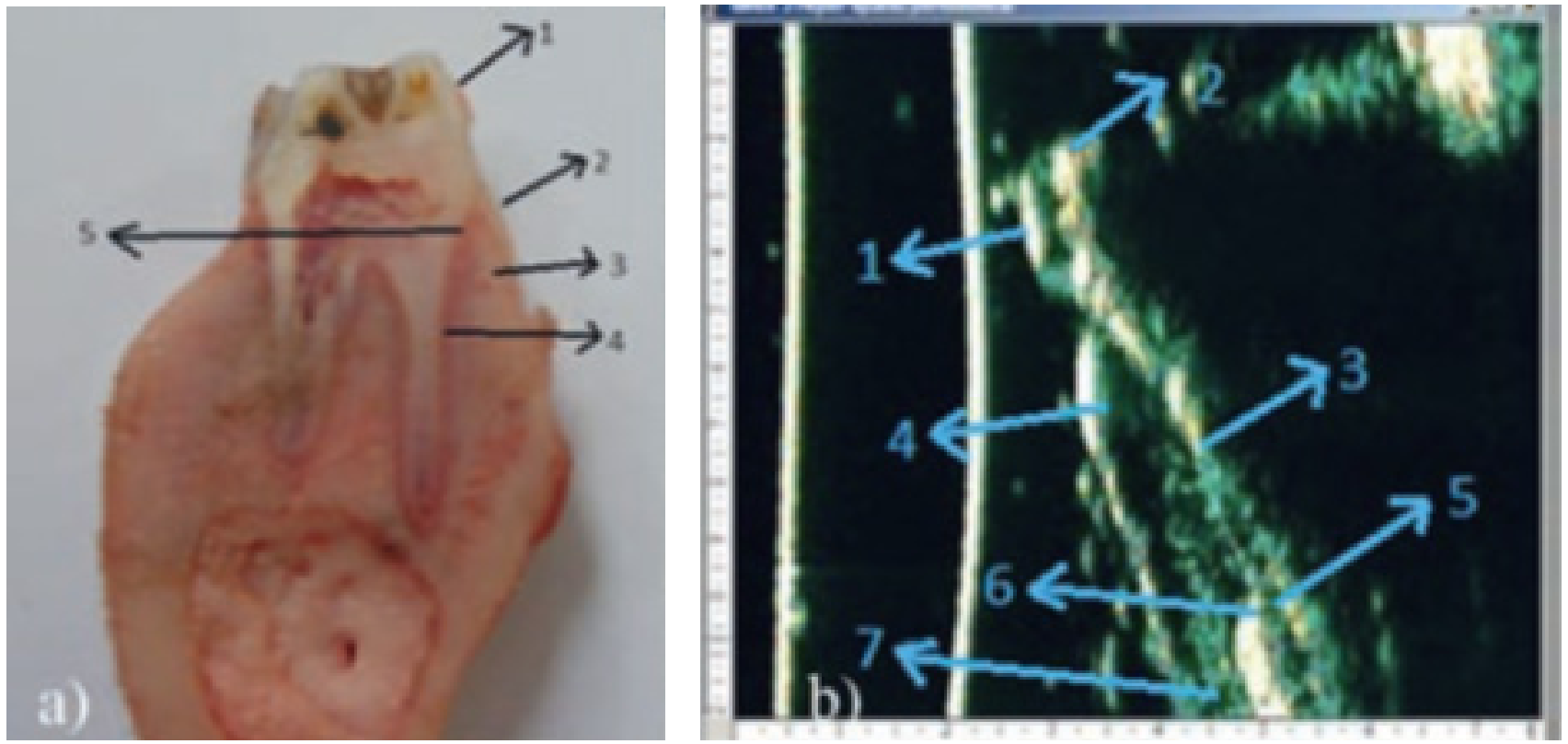

Figure 6. a) Sagittal section through a tooth: 1- glued gutta-percha landmark, 2- gingival margin, 3- cortical bone, 4- periodontal space, 5- tooth wall covered by cementum. b) DermaScan sagittal section through a tooth, visualized elements: 1- glued guttapercha-landmark, 2- crown enamel, 3- enamel-cement junction, 4- gingival margin, 5- tooth root, periodontal space, 6- cortical bone (coronal edge), 7- fix mucosa. 

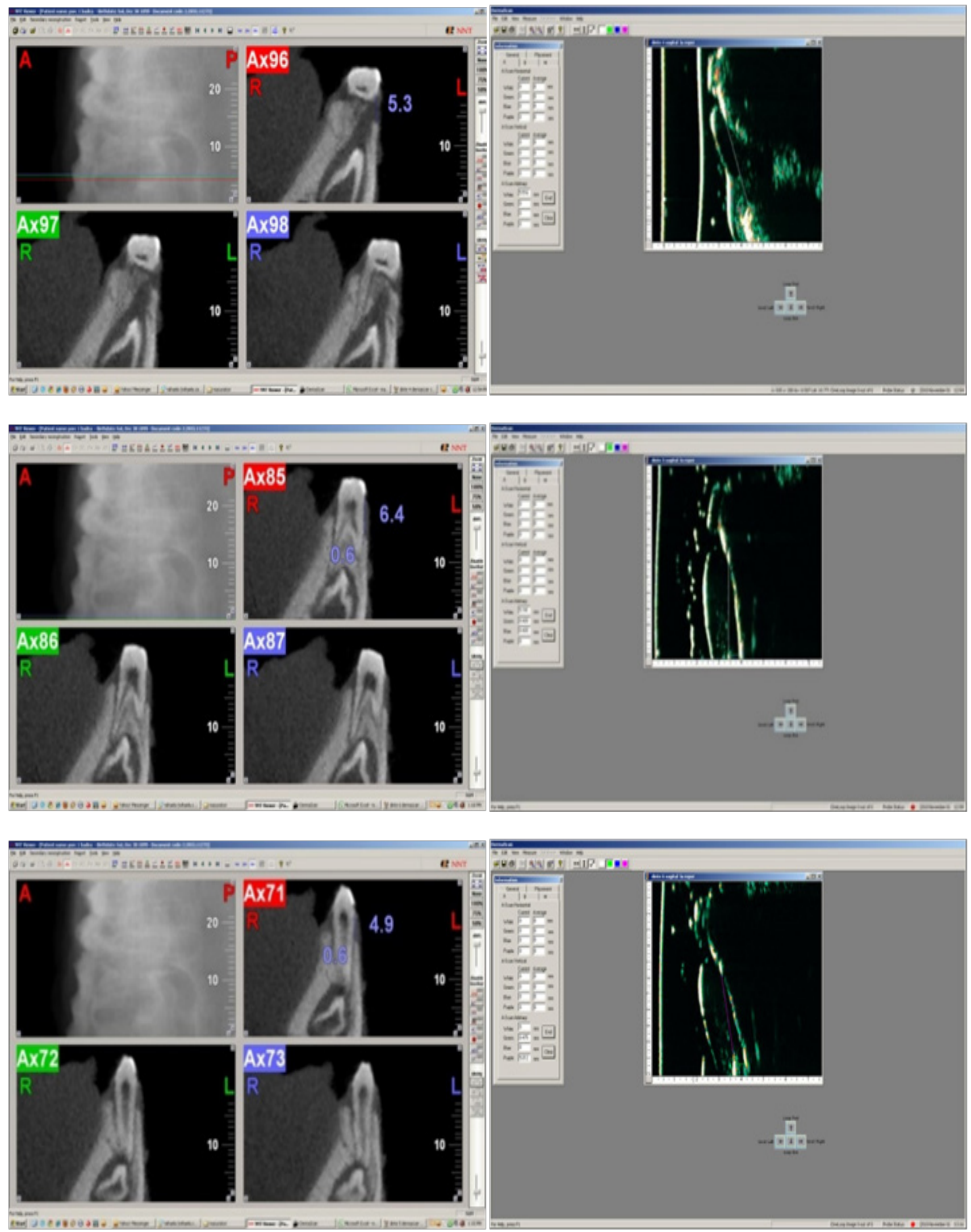

Figures 7-12. CBCT scan images compared with DermaScan images on the same sections [9]. 
Anatomical similarities of hard tissue can be observed on the same sections of the examined pig mandibles using X-ray CBCT imaging techniques and $20 \mathrm{MHz}$ periodontal ultrasonography (Figure 7-12). The data from the current study showed that the periodontal ultrasound examination may be a reliable method to assess the periodontal system because the measurements made in $\mathrm{B}$ mode have a high correlation with microscopy measurements, which is the gold standard. The cementum-enamel junction may be identified on ultrasonic images with high accuracy according to the tooth anatomical convexities. Therefore, this method could be used in the future for monitoring the periodontium if the size of the transducer permits it [9].

Identifying the periodontal pocket could be difficult most of the time (Figure 13). For this reason, using 2D image processing algorithms and increasing the ultrasound frequency could be a solution (Figure 14). Computer-assisted identification of the gingival sulcus and the periodontal epithelial junction on high-frequency ultrasound images lies in the identification and visualization of the gingival sulcus on US images, without the need of a periodontal probe that could influence the gingival attachment level. Moreover, the computer algorithm used in the analysis significantly increased the accuracy of the measurements, as confirmed by the fact that the values did not significantly differ from those obtained by microscopy $4 \mathrm{X}$ magnification as a gold standard of the same section as the ultrasonographic one [10].

Increasing the ultrasound frequency resulted in the improvement of the resolution of the obtained image (Figure 15).
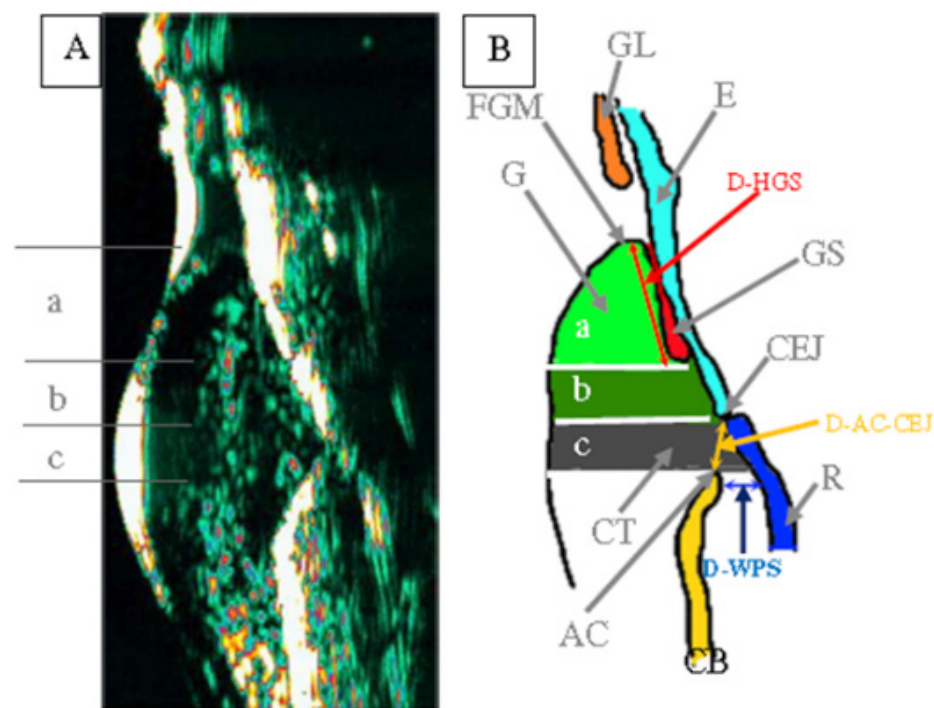

Figure 13. Periodontal ultrasonography using 20-MHz DermaScan C Cortex Technology: a) Periodontal ultrasonography using 20-MHZ DermaScan C Cortex Technology; b) Explanatory drawing: gutta percha landmark (GL), enamel tooth crown (E), gingival sulcus (GS), cementum-enamel junction (CEJ), tooth root (R), aleveolar crest (AC), connective tissue (CT), gingival tissue (G), free gingival margin (FGM), cortical bone (CB); a-histological sulcus, b- epithelial attachment, c- connective tissue attachment, biological width (b+c) [10].
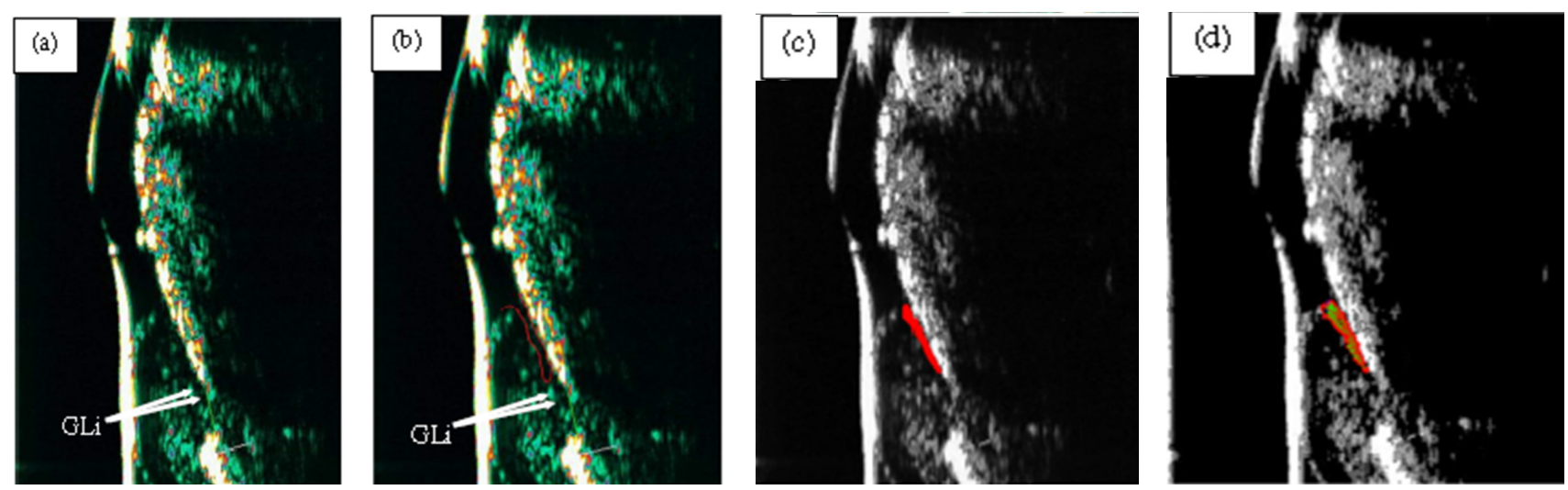

Figure 14. Periodontal ultrasonography using 20-MHz DermaScan C Cortex Technology. Computer generation of a gingival sulcus contour: a) Periodontal ultrasonography using 20-MHz DermaScan C Cortex Technology without gingival contour. The gingival ligaments are attached to the tooth (GLi); b) The real contour as traced by the medical expert; c) The final contour detected on the grayscale image and d) After the application of k-means clustering $(\mathrm{k}=4)[10]$. 


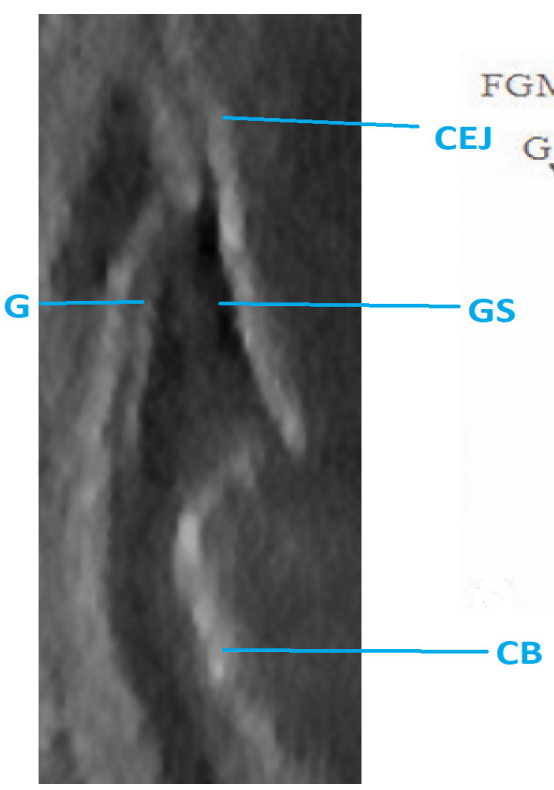

a.

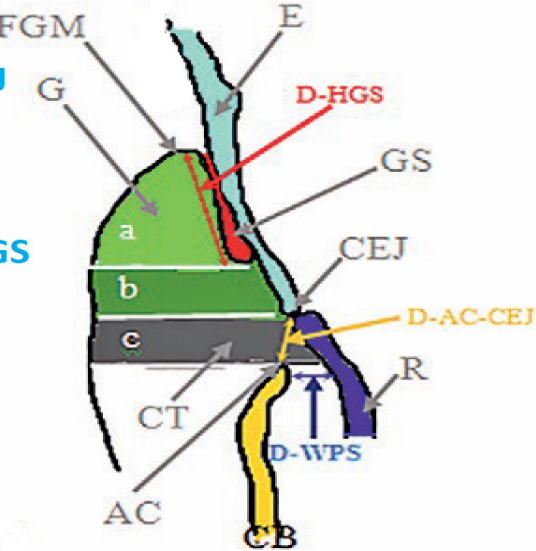

b.

Figure 15. a. periodontal ultrasonography using 23-MHZ Vinno6; 37 yars old, tooth 2.4, chronic periodontitis.

b. Explanatory drawing: enamel tooth crown (E), gingival sulcus (GS), cementum-enamel junction (CEJ), tooth root (R), alveolar crest $(\mathrm{AC})$, connective tissue (CT), gingival tissue $(\mathrm{G})$, free gingival margin (FGM), cortical bone (CB); a- histological sulcus, b- epithelial attachment, c- connective tissue attachment, biological width $(b+c)[10]$.

\section{Measuring and characterization of the dento- alveolar system using high frequencies ultrasound techniques. In vivo studies \\ The identification of the anatomical elements necessary for periodontal diagnosis, on $40 \mathrm{MHz}$ periodontal ultrasonography, was performed with success}

(Figure 16). All the anatomical elements were successfully identified using $40 \mathrm{MHz}$ periodontal ultrasonography with a $2 \mathrm{D}$ linear $1.2 \mathrm{~cm}$ footprint transducer. The examinations were performed transcutaneous, placing the transducer extra orally on the skin of the patient (Figure 17) [11].

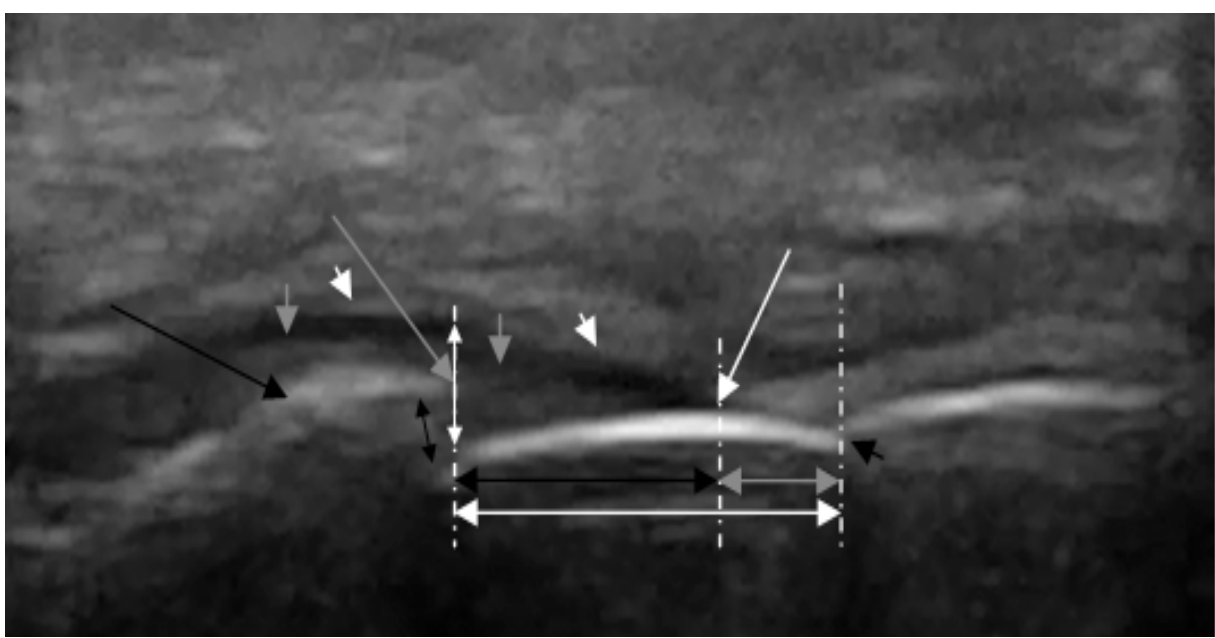

Figure 16. Ultrasound examination $40 \mathrm{MHz}$, longitudinal section, buccal surface, on tooth axis. Gingival epithelium (short white arrow), hypoechogenic connective tissue (short gray arrow), hyperechogenic cortical bone (long black arrow), cement-enamel junction (short black arrow), free gingival margin (long white arrow), alveolar crest (long gray arrow), gingival recession (UGR) distance between the cement-enamel junction and the gingival free margin (gray double arrow), UBJ distance between alveolar crest and cement-enamel junction (white long double arrow), UWPS periodontal space width (black short double arrow), UBG distance between the alveolar crest and free gingival margin (long black double arrow), UGW attached gingiva width to the alveolar crest limit (white short double arrow) [11]. 


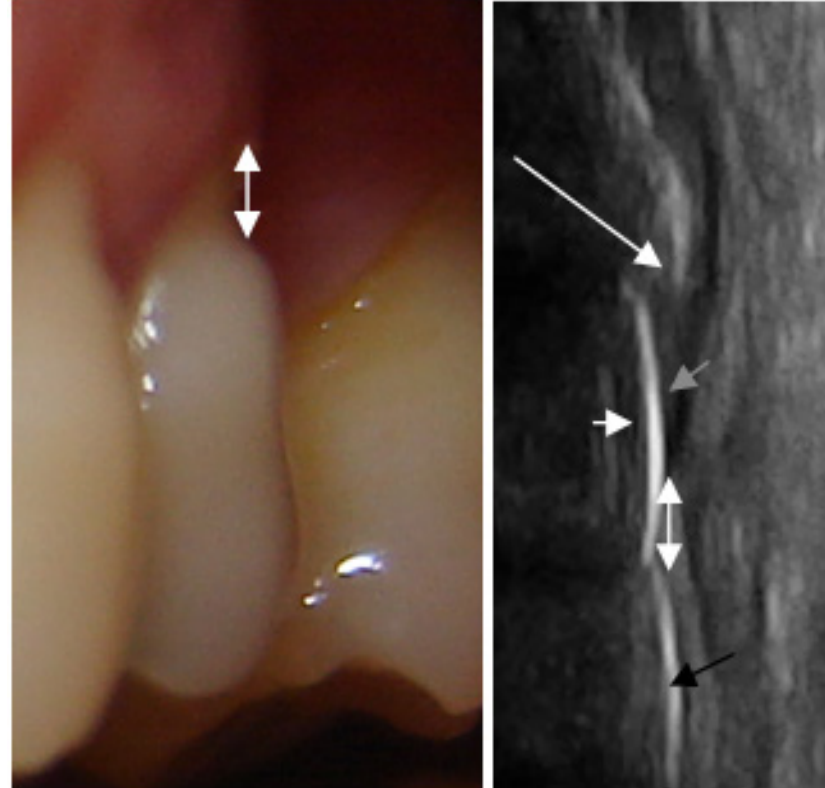

Figure 17. Gingival recession measured clinically and on ultrasound $40 \mathrm{MHz}$ : tooth crown (black arrow), tooth root (short white arrow), alveolar crest (long white arrow), gingival tissue (gray arrow), gingival recession (white double arrow). The gingival recession measured clinically compared with the same distance on the ultrasonographical images showed that the periodontal ultrasonography performed with $40 \mathrm{MHz}$ is a reliable imaging technique for qualitative and quantitative evaluation of the periodontal system [11].

Further in vivo studies led to the development of a new method of volumetric evaluation of the gingival inflammation (Figures 18,19). The $40 \mathrm{MHz}$ periodontal ultrasonography proved to be a reliable technique in the assessment of the gingival inflammation evolution following professional teeth cleaning. Nowadays the evaluation of the inflammation is assessed during the clinical examination visually and by the evaluation of the bleeding performed during the periodontal probing. These classifications are operator dependent and not very accurate. It is mandatory to develop an imaging technique for quantifying the volumetric changes of the gingival tissue with micrometric precision [12] and for the assessment of the inflammation degree in the musculoskeletal system, the examined tissue, based on the detection of non-specific extracellular modifications by ultrasound examinations [13].

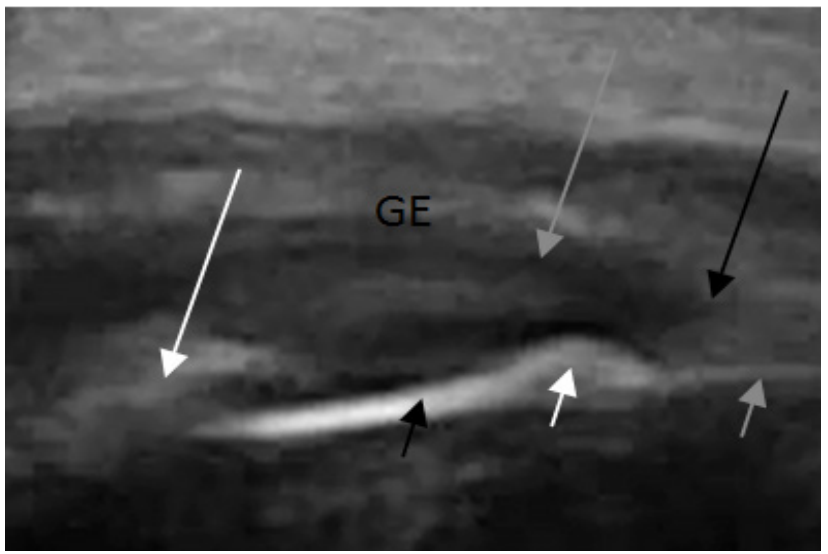

Figure 18. $40 \mathrm{MHz}$ ultrasound examination tooth 4.3 before scaling. The cortical bone (long white arrow), gingival connective tissue (long grey arrow), free gingival margin (long black arrow), root surface (short black arrow), subgingival calculus (short white arrow), tooth enamel (short grey arrow), gingival epithelium (GE) are evident [12].

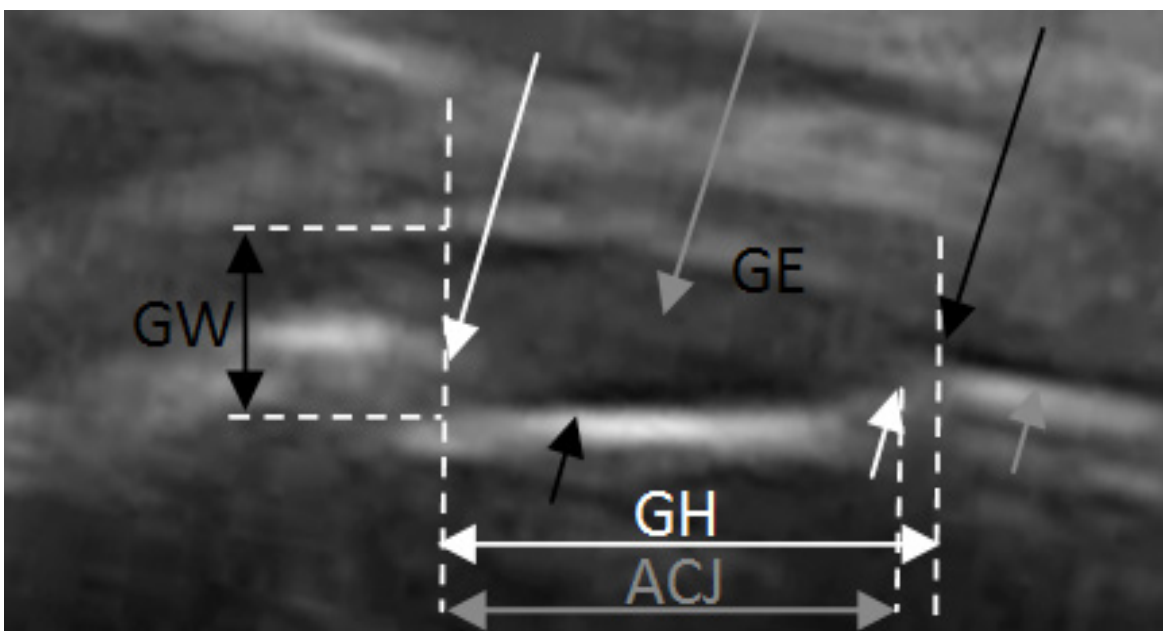

Figure 19. Ultrasound examination $40 \mathrm{MHz}$, longitudinal section, buccal surface, on tooth axis. Gingival epithelium (short white arrow), hypoechogenic connective tissue (short gray arrow), hyperechogenic cortical bone (long black arrow), cement-enamel junction (short black arrow), free gingival margin (long white arrow), alveolar crest (long gray arrow), gingival recession (UGR) distance between the cement-enamel junction and the gingival free margin (gray double arrow), UBJ distance between the alveolar crest and the cementenamel junction (white long double arrow), UWPS periodontal space width (black short double arrow), UBG distance between the alveolar crest and free gingival margin (long black double arrow), UGW attached gingiva width to the alveolar crest limit (white short double arrow) [11]. 
The transducer of Dermascan has $20 \mathrm{~mm}$ footprint. It was applied directly onto the alveolar process of the pig mandible using echographic gel for better results. Using a device like Dermascan, having a very large transducer (figure 1) makes impossible in vivo clinical studies. In order to perform highly efficient ultrasonographic imagistic investigations, an appropriate size of the transducer, correlated to the anatomy of the examined areas is needed. Thus, if small areas, difficult to be accessed need to be examined, then the transducer size need to be correlated to them.

Most of the in vivo studies which demonstrate the usefulness and the high accuracy of the ultrasonographic periodontal investigations were performed with standard transducers and for that reason the teeth were examined only on the buccal surface, directly applied onto the alveolar process, by intraoral approach or having a percutaneous trans genial approach [14]. There are studies which reported the intraoral ultrasound examination using an angled intraoral transducer resulting in visualizing the lingual nerve and the greater palatine foramen but not the periodontal tissue. The periodontal tissue was visualized only buccally by ultrasound intra- and extraoral examinations [15].

On buccal surfaces, it is quite easy to obtain the necessary information for periodontal diagnosis, whereas on the lingual surfaces, if the size of future transducers is better adapted, the necessary information will very likely be obtained. For now, with the existing ultrasound devices, it is difficult, or even impossible, to examine the periodontal tissues on proximal surfaces [16].

This is the reason why some researchers tried to develop custom ultrasonographic probes to carry out in vivo studies regarding ultrasonographic periodontal assessment.

\section{Bidimensional periodontal ultrasonographic investigations, using custom ultrasound probes}

The importance of the development of a specific ultrasound probe dedicated to intraoral examination, especially for periodontal ultrasonography, is highlighted by the results. Overall, if the probe can be positioned properly (more than $92 \%$ of cases), the distinction between soft and mineralized tissues is clear [17]. Salmon et al. managed to scan in vivo buccal surfaces and oral surfaces of the teeth [17].

Salmon et al. concluded that as it is atraumatic and without ionizing radiation, ultrasonography may frequently be repeated to monitor the stability of therapeutic interventions (mucogingival plastic surgery, marginal bone loss at peri-implant sites) dynamically and in real time, quantitatively and qualitatively. Ultrasonography offers new prospects for periodontal phenotyping (gum thickness), prevention (earlier detection of a small anatomic change), diagnosis and therapeutic monitoring of periodontal diseases and oral mucosa lesions. The periodontal biological width is directly accessible and measurable [17].
A much smaller probe was developed, a periodontal probe, as a spin-off of NASA technology. An ultrasonic transducer projects high frequency $(10-15 \mathrm{MHz})$ ultrasonic energy between the tooth and the gum and detects echoes of the returning wave. In the usual practice of ultrasonography, the time delay of the reflection is converted to a distance measurement by using the speed of sound in water (1482 $\mathrm{m} / \mathrm{s}$ ). The results from the classification scheme can identify ultrasonographic periodontal probe measurements [18].

The main characteristics of the specific ultrasound probes, developed by Salmon et al, for dentistry are:

- the reduced dimensions of the tip (Salmon et al. developed a probe having dimensions of the pseudocylindrical transducer: externally active diameter $3.6 \mathrm{~mm}$; overall dimensions: diameter $5 \mathrm{~mm}$; height 4.2 $\mathrm{mm}$, Radius of curvature (front face) $8 \mathrm{~mm}$. Bertoncini et al. developed a probe that had a miniaturized tip that could enter the periodontal pocket) [17];

- the angled shape, like the dentistry instruments from the examination kit or manual scaling $[17,18]$;

- high frequencies ultrasound, between $14-25 \mathrm{MHz}$ [15,17-19].

\section{Conclusions about 2D periodontal ultrasonography}

Even if the ultrasound waves cannot penetrate the cortical bone with thickness more than $1.1 \mathrm{~mm}$ working between $6-12 \mathrm{MHz}$ with a 40 -millimeter, linear probe [20], the periodontal ultrasonography is a reliable technique for visualizing the anatomical elements necessary to diagnose the periodontal status. Other recent studies show that US has high potential to supplement CBCT in measuring the cortical bone thickness [21], especially if the ultrasound scans are performed with a 3D imaging setup.

Ultrasonography has great potential to become a noninvasive diagnostic imaging tool for quantitative assessment of the periodontal structures and better delivery of oral care. [19] Ultrasound has significant potential to aid surgeons in "visualizing" oral structures during minimally invasive surgery and guide local anesthesia for difficult cases [15] due to the fact that it is a real time imaging examination.

Using this non-invasive imaging technique one can see the dentino-enamel junction, the cortical bone, the radicular surface from the crown to the alveolar bone, the gingival tissue without interfering with those elements during the examination. Calculus visualization is possible before and after scaling in order to appreciate the quality of the treatment.

\section{Advantages, disadvantages and applications of ultrasonography in the dento-maxillofacial region}

Disadvantages of US: teratogenic effects with very low probability; metallic implants, dental fillings and restorations may cause blurring of the image; the articular disk is difficult to visualize with US when it is placed between 
two hard tissue structures; in the case of acute conditions with facial edema and empyema bone visualization may be complicated $[22,23]$.

Advantages: real time, portable, inexpensive, radiation free, and noninvasive imaging method making use of US as an important diagnostic aid for maxillofacial surgeons to diagnose the pathologies related to this region especially in pediatrics and pregnant women where X-rays are contraindicated [24].

Application of US in the dentomaxillofacial region: cervical lymphadenopathy, swelling in the orofacial region, space infection, vascular malformations, salivary gland pathology, periodontal US is a non-invasive diagnostic method for measuring pocket depth which is an indicator of periodontal health, periodontal bony defects, monitoring anatomical changes like periodontal ligament space and free gingiva during orthodontic treatment, assessment of bony cortical plates, detection of carious lesions, soft tissue lesions, measurement of muscle and gingival thickness, diagnosis of temporomandibular disorders, implant dentistry and dental scanning, salivary glands and ducts, as well as the mouth floor, the buccal, labial, and palatal mucosa, the tongue, soft tissues, glands, metastasis and ultrasound imaging of hard tissues (fractures, bone healing, dental fractures or cracks, osteomyelitis, visualizing important landmarks not only in implant dentistry but also to oral maxillofacial surgeons like mental foramen, foreign body detection [16,22-27].

Not using ultrasound imaging in dentistry as a common investigation method may be because of a very difficult reproducibility of the $2 \mathrm{D}$ ultrasonographic slices in order to have the possibility to compare different investigations efficiently and that is why the method is still operator dependent. For an ultrasound investigator at least a 2- month practice period is required in order for them to achieve acceptable performance and a minimum of 10 months to have the skills to perform good musculoskeletal ultrasonography [28]. After comparing a computed tomography with musculoskeletal ultrasonography, the conclusion was that after the parameters were set, the tomography was not operator-dependent, but it was not suited for daily practice at the office because it is an imaging technique with rather X-ray exposure. The musculoskeletal ultrasonography can scan several joints on the same visit, it can scan the blood flow at the same time, but many authors view the musculoskeletal ultrasound as an operator-dependent technique that should be overcome [29].

The problems to be solved and the limitations regarding the periodontal ultrasonography were that the operator-dependence was still very important due to the difficulty encountered in obtaining a certain section generated by the incidence of the transducer and of the ultrasonografic plane at the level of the examined area. This incidence plane is very important in obtaining a high quality ultrasonographic image. $2 \mathrm{D}$ ultrasonography also depends on the size of the transducers most of the time making it difficult to be applied especially intraorally if it is not a miniaturized transducer. On the other hand, using a miniaturized transducer can become time consuming and very difficult to be used because the operator must identify the anatomic elements necessary for making the diagnosis in a very small field of view. A proper balance should be found between the size of the transducer and the anatomy of the examined area. If the transducer footprint is large, then the anatomical elements can be easily captured in the same image, but especially because of the curvatures of the maxilla and of the mandible, the quality of the bidimensional ultrasonographic images is very low. Because of the curvatures of the alveolar processes a smaller transducer will offer a better contact with the ultrasound scanned area and for that reason the operator can obtain a higher quality of the ultrasonographic image. If one is working with a bidimensional imaging technique it is mandatory that all the diagnostic elements be present in the same bidimensional image captured. If not, those bidimensional images cannot be composed and the information is useless. The alternative to this issue could be moving to $3 \mathrm{D}$ examinations.

Using 2D ultrasonography is time consuming and requires extensive experience in the field and that is probably the reason why $2 \mathrm{D}$ ultrasound imaging in dentistry is not used on a regular basis. 3D ultrasonographic reconstructions could be a very good alternative to eliminate the operator dependence, especially for the periodontal examination to replace the periodontal probing (measuring the periodontal pockets depth by using a periodontal probe).

\section{Digital dentistry era - 3D examinations}

Digital Smile Design is a method that allows us to digitally design the smile of our patients, by obtaining a simulation and pre-visualization of the therapeutic result [30]. Periodontal surgery associated with prior waxing, mock-up, and the use of digital tools to design the smile is the current trend of reverse planning in periodontal plastic surgery [31]. Cosmetic dentistry is the integration of all the areas of dentistry; Digital Smile Design programs are used for objective esthetic analysis and virtual treatment planning by editing photographs and/or scanned models of patients [32]. The last decade has seen an increasing number of optical intraoral scanner devices, and these are based on different technologies; the choice of which may impact on their clinical use [33].

Question: How is it possible to have a correct pre-visualization of the therapeutic result and to know the effects on the long run of the treatments if we do not take into consideration the information about the gingival epithelial insertion, about the biological width, the anatomical interference in the deeper layers of the superficial periodontal tissue? 


\section{The missing link, a soft tissue 3D scanner}

Motivation: The current standard of care for measuring periodontal pockets is periodontal probing, an analogue technology in a digital age [34]. For intraoral scanners it can be difficult to detect deep margin lines in prepared teeth and/or in case of bleeding [35]. The main side effect of this inconvenience is reflected on the periodontal tissue, generating inflammation and a possible cause of an early prosthetic failure.

The solution: Marroti et al. performed an in vitro study showing that because of its ability to capture hard structures behind soft tissue, ultrasound-based microscanning may be a promising alternative for taking digital impressions of teeth, especially in the case of subgingival margin preparations [36]. This study showed no data about periodontal pockets, gingival epithelial insertion.

Mahmoud et al. have developed a 3D ultrasound scanning technique since 2010, and they successfully performed 3D ultrasound modeling of bony defects [37]. Also an in vitro study with no data about soft tissue scans, periodontal pockets, gingival epithelial insertion, but like the study of Marotti et al. presented the true potential of 3D ultrasonography.

Using photoacoustic ultrasound Lin et al. performed high-spatial resolution imaging of probing depths on an experimental model using pig mandibles. Specific contrast from dental pockets was achieved with food-grade cuttlefish ink as a contrast medium [38].

In 2018 Moore et al. reported the first use of photoacoustic imaging for monitoring periodontal health in humans by quantitating pocket depths and generating 3D modeling of the periodontal tissue [39].

The advantages of a 3D free hand ultrasound scanner

The freehand method has advantages over the use of a 3D US transducer like lower cost, higher volume quality and improved field of view [40]. Bidimensional transducers already on the market can be used for 3D ultrasound scanning having proper 3D modeling techniques (Figures 20, 21,22).

3D ultrasound scanner reconstruction - using 2D $23 \mathrm{MHz}$ transducer and a spatial positioning sensor

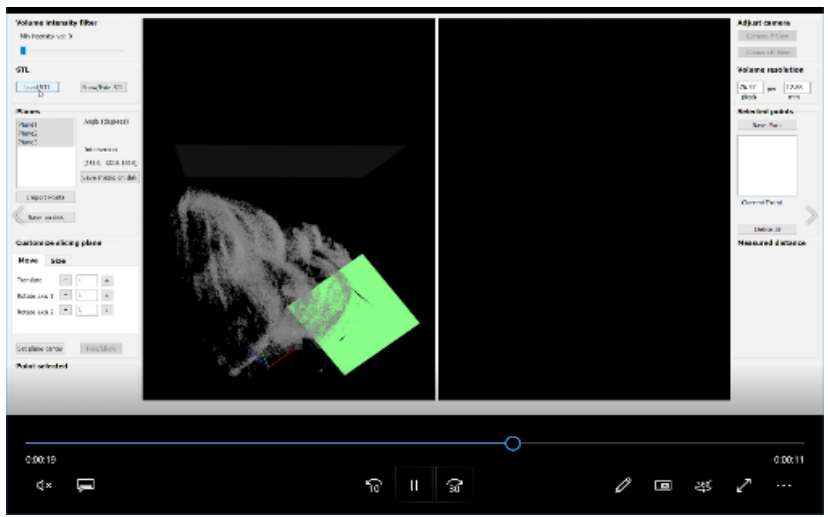

Figure 20. 3D ultrasonographic point cloud of a lateral maxillary region.

The 3D ultrasound model can be visualized, sectioned, measured, and compared over time with other 3D models.

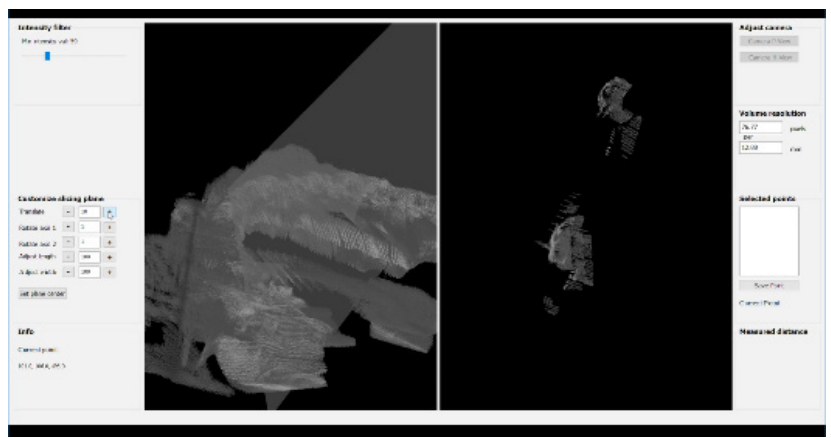

Figure 21. Cross-section through an ultrasonographic point cloud.
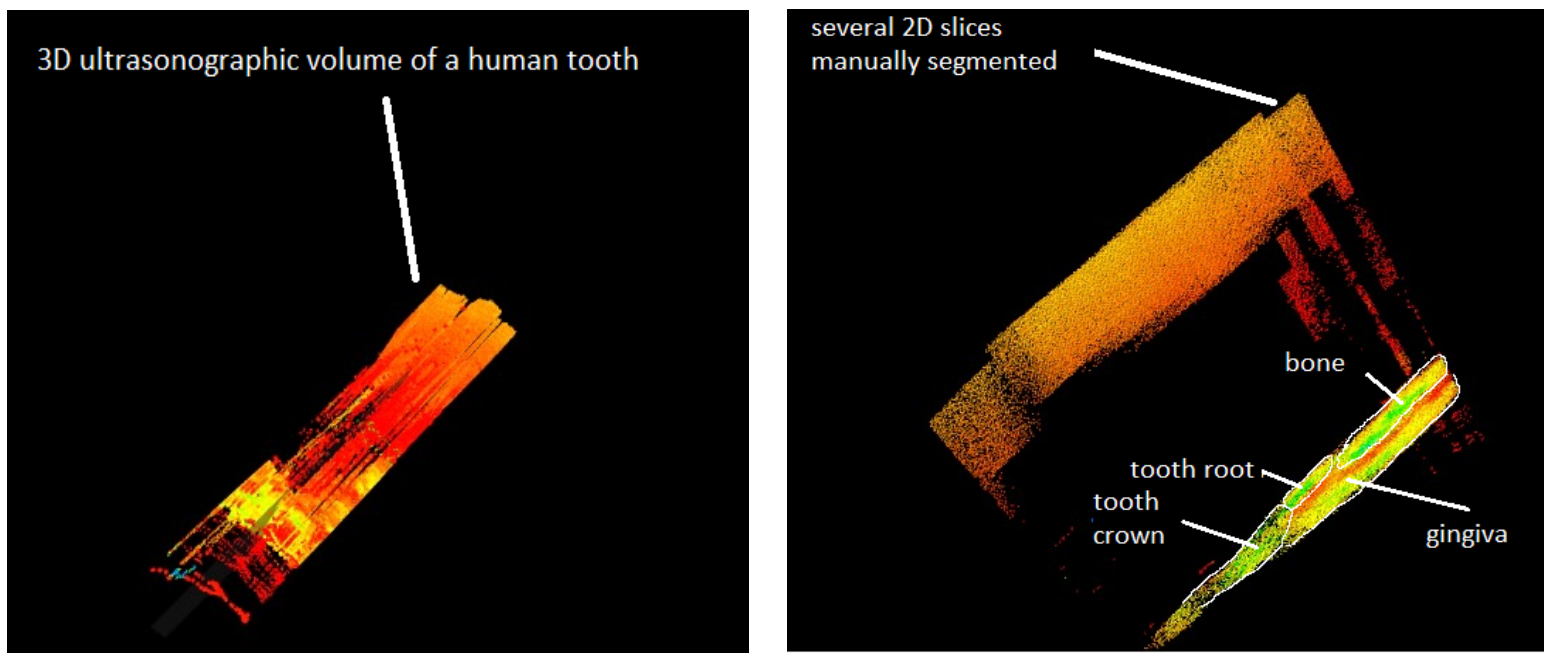

Figure 22. 2D section through 3D ultrasound scan: Maxillary periodontal tissue - canine region. 
One can see that the periodontal ultrasonographic 3D model was cross sectioned with an arbitrary plane chosen by the operator and the information from several frames was taken into consideration for diagnosis (Figure 22).

The main advantages of the 3-D domain for US systems in comparison to 2-D images: visualization of the entire structure of an organ in three dimensions, less dependence on an operator, orientation-independent visualization, measurement of quantitative attributes in three dimensions, repeatability of ROI examination, fusion with other 3-D image modalities [40].

The advantage of 3D volume dataset allows periodontists and orthodontists to visualize the alveolar structures on the lingual and buccal sides, which cannot be visualized on a $2 \mathrm{D}$ radiograph. Currently, the use of CBCT is commonly restricted to the assessment of the hard tissues such as bone, tooth, implant or dry skull due to poor soft tissue contrast [41]. The three-dimensional imaging has made the complex cranio-facial structures more accessible for examination and early and accurate diagnosis of deep-seated lesions [42]. The last decade has shown an abundance of promising research attacking tissue anisotropy and complex geometries through simulation and modeling methods. Some technologies are being tested in clinical situations, and consequently it may not be long before ultrasonographic methods are common acts in the dental office. Indeed, as ultrasound and associated computer technology continue to shrink in size and cost, many new opportunities for diagnostic ultrasound may arise in dentistry [43].

Making the technique less user-dependent by progressing from a 2D ultrasonographic imagistic technique to a 3D examination will allow ultrasonography to be widely used for non-invasive and cost-effective scanning of soft-tissues and of hard-tissues surfaces.

\section{Acknowledgements}

This study was partially realized with the material and logistic support, namely necessary technology and equipment, of Chifor Research SRL, through the project Periodontal ultrasonography in diagnosing and monitoring the periodontal disease - Chifor Research SRL, Operational Programme Competitivity, Ministry of European Funds, P_38_930\12.10.2017. The authors Radu Chifor, Mindra Eugenia Badea, Ioana Chifor, Alexandru Florin Badea would like to extend their thanks to Chifor Research SRL for their financial support of this research within the above-mentioned project.

\section{References}

1. Petersen PE, Ogawa H. Strengthening the prevention of periodontal disease: the WHO approach. J Periodontol. 2005;76:2187-2193.
2. Lang NP, Schätzle MA, Löe H. Gingivitis as a risk factor in periodontal disease. J Clin Periodontol. 2009;36 Suppl 10:38.

3. Larjava H, Koivisto H, Häkkinen L, Heino J. Epithelial integrins with special reference to oral epithelia. J Dent Res. 2011;90:1367-1376.

4. Preshaw PM. Detection and diagnosis of periodontal conditions amenable to prevention. BMC Oral Health. 2015;15 Suppl 1:S5. doi: 10.1186/1472-6831-15-S1-S5.

5. Highfield J. Diagnosis and classification of periodontal disease. Aust Dent J. 2009;54 Suppl 1:S11-S26.

6. Deepa R, Prakash S. Accuracy of probing attachment levels using a new computerized cemento-enamel junction probe. J Indian Soc Periodontol. 2012;16:74-79.

7. Newman M, Takei H, Klokkevold P, Carranza F. Carranza's Clinical Periodontology. Tenth Edition. Saunders, Elsevier, 2006: pp. 445, 1224.

8. Chifor R, Badea ME, Hedesiu M, Serbanescu A, Badea AF. Experimental model for measuring and characterisation of the dento-alveolar system using high frequencies ultrasound techniques. Med Ultrason. 2010;12:127-132.

9. Chifor R, Hedeşiu M, Bolfa P, Catoi C, Crişan M, Serbănescu A. The evaluation of $20 \mathrm{MHz}$ ultrasonography, computed tomography scans as compared to direct microscopy for periodontal system assessment. Med Ultrason. 2011;13:120126.

10. Chifor R, Badea ME, Mitrea DA, Badea IC, Crisan M, Chifor I. Computer-assisted identification of the gingival sulcus and periodontal epithelial junction on high-frequency ultrasound images. Med Ultrason. 2015;17:273-279.

11. Chifor R, Badea ME, Hedesiu M, Chifor I. Identification of the anatomical elements used in periodontal diagnosis on 40 mhz periodontal ultrasonography. Rom J Morphol Embryol. 2015;56:149-153.

12. Chifor R, Badea ME, Vesa SC, Chifor I. The utility of 40 $\mathrm{MHz}$ periodontal ultrasonography in the assessment of gingival inflammation evolution following professional teeth cleaning. Med Ultrason. 2015;17:34-38.

13. Bierry G, Dietemann JL. Imaging evaluation of inflammation in the musculoskeletal system: current concepts and perspectives. Skeletal Radiol. 2013;42:1347-1359.

14. Zimbran A, Dudea S, Dudea D. Evaluation of periodontal tissues using $40 \mathrm{Mhz}$ ultrasonography. Preliminary report. Med Ultrason. 2013;15:6-9

15. Chan HL, Wang HL, Fowlkes JB, Giannobile WV, Kripfgans OD. Non-ionizing real-time ultrasonography in implant and oral surgery: A feasibility study. Clin Oral Implants Res. 2017;28:341-347.

16. Caglayan F, Bayrakdar IS. The Intraoral Ultrasonography in Dentistry. Niger J Clin Pract. 2018;21:125-133.

17. Salmon B, Le Denmat D. Intraoral ultrasonography: development of a specific high-frequency probe and clinical pilot study. Clin Oral Investig. 2012;16:643-649.

18. Bertoncini CA, Hinders MK. An ultrasonographic periodontal probe. Review of quantitative nondestructive evaluation, vol. 29, American Institute of Physics, 2010: pp. 
1566-1572.

19. Nguyen KT, Le LH, Kaipatur NR, Zheng R, Lou EH, Major PW. High-Resolution Ultrasonic Imaging of DentoPeriodontal Tissues Using a Multi-Element Phased Array System. Ann Biomed Eng. 2016;44:2874-2886.

20. Adibi S, Shakibafard A, Karimi Sarvestani Z, Saadat N, Khojastepour L. Effect of Cortical Bone Thickness on Detection of Intraosseous Lesions by Ultrasonography. Radiol Res Pract. 2015;2015:797593.

21. Degen K, Habor D, Radermacher K, Heger S, Kern JS, Wolfart $\mathrm{S}$, et al. Assessment of cortical bone thickness using ultrasound. Clin Oral Implants Res. 2017;28:520-528.

22. Evirgen Ş, Kamburoğlu K. Review on the applications of ultrasonography in dentomaxillofacial region. World $\mathrm{J}$ Radiol. 2016;8:50-58.

23. Marotti J, Heger S, Tinschert J, Tortamano P, Chuembou $\mathrm{F}$, Radermacher $\mathrm{K}$, et al. Recent advances of ultrasound imaging in dentistry - a review of the literature. Oral Surg Oral Med Oral Pathol Oral Radiol. 2013,115:819-832.

24. Rama Mohan K, Koteswara Rao N, Leela Krishna G, Santosh Kumar V, Ranganath N, Vijaya Lakshmi U. Role of ultrasonography in oral and maxillofacial surgery: a review of literature. J Maxillofac Oral Surg. 2015;14:162-170.

25. Laher AE, Wells M. Ultrasonographically locating the mental foramen and its soft tissue relations. Dentomaxillofac Radiol. 2016;45:20160236.

26. Zimbran A, Dudea D, Gasparik C, Dudea S. Ultrasonographic evaluation of periodontal changes during orthodontic tooth movement - work in progress. Clujul Med. 2017;90:93-98.

27. Gad K, Ellabban M, Sciubba J. Utility of Transfacial Dental Ultrasonography in Evaluation of Cystic Jaw Lesions. J Ultrasound Med. 2018;37:635-644.

28. Ohrndorf S, Naumann L, Grundey J, Scheel T, Scheel $\mathrm{AK}$, Werner $\mathrm{C}$, et al. Is musculoskeletal ultrasonography an operator-dependent method or a fast and reliably teachable diagnostic tool? Interreader agreements of three ultrasonographers with different training levels. Int $\mathrm{J}$ Rheumatol. 2010;2010:164518.

29. Checa A. Ultrasonography, an operator-dependent modality versus dual-energy computed tomography (DECT) in the detection of chondrocalcinosis: with regard to Tanikawa et al.'s study. J Orthop Surg Res. 2018;13:255.

30. Cervino G, Fiorillo L, Arzukanyan AV, Spagnuolo G, Cicciù M. Dental Restorative Digital Workflow: Digital Smile Design from Aesthetic to Function. Dent J (Basel). 2019;7. pii: E30.

31. Santos FR, Kamarowski SF, Lopez CAV, Storrer CLM, Neto AT, Deliberador TM. The use of the digital smile design concept as an auxiliary tool in periodontal plastic surgery. Dent Res J (Isfahan). 2017;14:158-161.

32. Omar D, Duarte C. The application of parameters for comprehensive smile esthetics by digital smile design programs: A review of literature. Saudi Dent J. 2018;30:7-12.

33. Richert R, Goujat A, Venet L, Viguie G, Viennot S, Robinson P, et al. Intraoral Scanner Technologies: A Review to Make a Successful Impression. J Healthc Eng. 2017;2017:8427595.

34. Elashiry M, Meghil MM, Arce RM, Cutler CW. From manual periodontal probing to digital 3-D imaging to endoscopic capillaroscopy: Recent advances in periodontal disease diagnosis. J Periodontal Res. 2019;54:1-9.

35. Mangano F, Gandolfi A, Luongo G, Logozzo S. Intraoral scanners in dentistry: a review of the current literature. BMC Oral Health. 2017;17:149.

36. Marotti J, Broeckmann J, Chuembou Pekam F, Praça L, Radermacher K, Wolfart S. Impression of Subgingival Dental Preparation Can Be Taken with Ultrasound. Ultrasound Med Biol. 2019;45:558-567.

37. Mahmoud AM, Ngan P, Crout R, Mukdadi OM. Highresolution 3D ultrasound jawbone surface imaging for diagnosis of periodontal bony defects: an in vitro study. Ann Biomed Eng. 2010;38:3409-3422.

38. Lin CY, Chen F, Hariri A, Chen CJ, Wilder-Smith P, Takesh $\mathrm{T}$, et al. Photoacoustic Imaging for Noninvasive Periodontal Probing Depth Measurements. J Dent Res. 2018;97:23-30.

39. Moore C, Bai Y, Hariri A, Sanchez JB, Lin CY, Koka S, et al. Photoacoustic imaging for monitoring periodontal health: A first human study. Photoacoustics. 2018;12:67-74.

40. Mozaffari MH, Lee WS. Freehand 3-D Ultrasound Imaging: A Systematic Review. Ultrasound Med Biol. 2017;43:20992124.

41. Nguyen KT, Pachêco-Pereira C, Kaipatur NR, Cheung J, Major PW, Le LH. Comparison of ultrasound imaging and cone-beam computed tomography for examination of the alveolar bone level: A systematic review. PLoS One. 2018;13:e0200596. doi: 10.1371/journal.pone.0200596.

42. Shah N, Bansal N, Logani A. Recent advances in imaging technologies in dentistry. World J Radiol. 2014;6:794-807.

43. Ghorayeb SR, Bertoncini CA, Hinders MK. Ultrasonography in dentistry. IEEE Trans Ultrason Ferroelectr Freq Control. 2008;55:1256-1266. 